\title{
Inhibition of glycogen synthase kinase-3-beta (GSK3 $\beta$ ) blocks nucleocapsid phosphorylation and SARS-CoV-2 replication
}

\section{Tirosh Shapira}

University of British Columbia

\section{Celine Rens}

University of British Columbia

\section{Virginia Pichler}

University of British Columbia

\section{William Rees}

University of British Columbia

\section{Theodor Steiner}

University of British Columbia

\section{Francois Jean}

University of British Columbia

\section{Dirk Winkler}

Kinexus Bioinformatics Corporation,

\section{Iqbal Sarai}

Kinexus Bioinformatics Corporation, https://orcid.org/0000-0001-7013-5742

\section{Steven Pelech}

University of British Columbia

Yossef Av-Gay ( $\square$ yossi@mail.ubc.ca )

University of British Columbia https://orcid.org/0000-0002-9547-0237

\section{Article}

Keywords:

Posted Date: January 7th, 2022

DOI: https://doi.org/10.21203/rs.3.rs-1197371/v1

License: (1) (1) This work is licensed under a Creative Commons Attribution 4.0 International License.

Read Full License 


\section{Abstract}

GSK3 $\beta$ has been proposed to have an essential role in Coronaviridae infection. Screening of a targeted library of GSK3 $\beta$ inhibitors against SARS-CoV-2 and HCoV-229E resulted in identification of high proportion of active compounds with low toxicity to host cells. A select lead compound, T-1686568, showed dose-dependent activity against SARS-CoV-2 transcription, translation and viral particle release in multiple cell lines and primary organoids. A protein kinase substrate profiling assay combined with western blot analysis showed that SARS-CoV-2 nucleocapsid is phosphorylated by GSK3ß on S180/S184, S190/S194 and T198 which have already been primed in the adjacent phospho-sites S188, T198 and S206 respectively. Inhibition by T-1686568 resulted in reduction of the S1 Spike protein levels, an accumulation of the Nucleocapsid (N) protein and maintenance of the non-structural (NSP2) level in infected Huh-7.5.1 cells, indicating that N phosphorylation might serve as a critical precursor for processing and release of mature viruses.

\section{Introduction}

Coronavirus disease 2019 (COVID-19) is caused by the novel coronavirus SARS-CoV-2, which has infected nearly 300 million people worldwide, killed over 5 million people, and caused significant global disruptions to social and economic structures [1,2]. Due to our limited knowledge of the molecular details of SARS-CoV-2 pathogenesis, the majority of current antiviral therapeutic efforts are focused on either repurposing existing antiviral drugs or finding new drugs that target viral entry or replication.

Most infected patients clear SARS-CoV-2 infection without treatment, through mounting innate and adaptive immune responses, demonstrating that human hosts have a built-in capacity to neutralize and overcome acute infections [3]. At the molecular level, surveillance and response to infection is mediated by various cellular signaling pathways that activate protective host processes including autophagy, apoptosis or even necrosis of infected cells. Consequently, we undertook a corresponding approach, examining the modulation of host signaling proteins that enhance the cell's innate ability to clear infection or may be important for viral replication.

Signaling mediated responses to infection rely on protein-protein interactions (PPIs) and associated posttranslational phosphorylation. A PPI study [4] analysing interactions between SARS-CoV-2 proteins and the human proteome identified over 300 high-confidence PPIs. Of the $300+$ human proteins identified, many are protein signaling targets of approved, investigational, or pre-clinical drugs [4]. These PPIs, therefore, are potential targets for the use of Host Directed Therapies (HDTs) that target host pathways and processes and not the virus directly. As viral infections hijack host functions, HDTs may interfere with these interactions or contribute to the host cell's ability to fight the infection. Additionally, as HDTs do not target the virus directly, there is greater potential for the HDTs to provide broader protection against various viral strains and alleviate pressures that cause drug resistance through viral mutations. 
Screening of a library of FDA-approved drugs for inhibitors of coronavirus replication identified Abelson (Abl) kinase inhibitors, including imatinib, as inhibitors of both the 2003 SARS-CoV strain and MERS-CoV in vitro [5]. Interestingly, inhibition of another key signaling protein, glycogen synthase kinase-3 (GSK3), was found to reduce viral nucleocapsid ( $\mathrm{N}$ protein) phosphorylation in SARS-CoV-infected VeroE6 cells and decreased viral titer and cytopathic effects. The effect of the GSK3 inhibitor was reproduced with another coronavirus, the neurotropic strain of mouse hepatitis virus. These results indicate that GSK3 is critical for coronavirus $\mathrm{N}$ protein phosphorylation and indicate that it plays a role in regulating the viral life cycle [6]. Furthermore, SARS-CoV-2 N protein phosphorylation, within an arginine- and serine-rich (RS) domain, was recently shown to be abolished in GSK3a and GSK3 $\beta$ knock-down cells [7]. As such, GSK3 $\beta$ has been suggested as a suitable candidate target for COVID-19 host-directed antiviral therapy [8].

GSK3 was first identified as a key control kinase of glycogen synthesis [9] and occurs in two distinct isoforms, designated GSK3a and GSK3 $\beta$ [10], each with differential regulation [11] and tissue expression [12]. GSK3 serves a key signaling role [13] in many regulatory processes through intersection with the $\mathrm{PI} 3 \mathrm{~K}, \mathrm{mTOR}, \mathrm{PKB} / \mathrm{AKT}, \mathrm{WNT}$, and MAPK pathways [14]. As such, GSK3 is targeted in drug discovery efforts in Alzheimer's disease, cancer, diabetes, multiple sclerosis, and others (reviewed in [15]). GSK3 $\beta$ has also been shown to be involved in the control of bacterial [16] and viral infections [7].

A recent study investigated the effect of kinase inhibitors against COVID-19 [7]. Enzastaurin, a clinically well-tolerated protein-serine/threonine kinase inhibitor, that is known to reduce GSK3 activity, was shown to reduce infection in the A549-ACE2 human lung cancer-derived cell line and Vero E6 cells. About 10mM were needed to inhibit $\mathrm{N}$ protein phosphorylation and SARS-CoV-2 replication in cells, whereas only a fraction, $1 \mathrm{mM}$, is required for GSK3 inhibition in humans [7]. Lithium chloride, a non-specific kinase inhibitor used to treat patients with bipolar disorder (BD), was shown to reduce the risk of developing COVID-19 compared to non-users [7]; however, current clinical therapeutic ranges have not been observed to be effective in vitro. Thus, although GSK3 has been confirmed to play a role in $\mathrm{N}$ protein phosphorylation for viral replication, given GSK3 inhibition varied across conditions, further screening is required to identify better compounds that are effective against this host derived therapeutic target.

In this study, we screened a GSK3 $\beta$-focused library of inhibitors against SARS-CoV-2 and human coronavirus (HCoV)-229E. Select compounds were validated in clinically-relevant immortalized cell line models, assessed for cytotoxic effects and antiviral activity was confirmed in patient-derived intestinal organoids.

\section{Results}

Screening of GSK3 $\beta$ focused library. A focused GSK3 $\beta$ inhibitor library [17] provided by Takeda Pharmaceutical Company (Japan) was screened against both SARS-CoV-2 and human alpha coronavirus HCoV-229E infected Huh-7.5.1 cells, monitoring either dsRNA or N protein levels as markers of infection (Supplementary Table 1). As seen in Figure 1, a high proportion of compounds active against GSK3 $\beta$ were effective in reducing SARS-CoV-2 and HCoV-229E infection. SARS-CoV-2 was initially probed 
using viral $\mathrm{N}$ protein, a marker of viral translation. Of 83 screened compounds, 7 compounds (8\%) showed $50 \%$ or greater inhibition, with nearly half $(n=40)$ of the compounds $(48 \%)$ having some inhibitory effect (>10\%) (Figure 1A).

We also probed for the presence of dsRNA, a marker of viral replication in RNA viruses. Screening for inhibition of $\mathrm{N}$ protein and dsRNA yielded similar readouts, (Supplementary figure 1 ) indicating that inhibition of $\mathrm{N}$ protein transcription is a reliable indicator of infection and of general viral load. For screening against HCoV-229E, we measured dsRNA (Figure 1A) and demonstrated that targeting GSK3 $\beta$ leads to a higher average level of inhibition over SARS-CoV-2, (31\% vs. $19 \%)$, in terms of number of active compounds that inhibit infection greater than $50 \%$. Together, the high ratio of potential hits and relatively low toxicity of the library as a whole support the viability of GSK3 $\beta$ as a target for HDT in COVID-19 and indicates that inactivation of GSK3 $\beta$ is a valid target for the use against coronaviruses.

Dose and cell dependent analysis of hit compounds. Next, three active compounds, T-1686568 (P0717632), P-7657632, and P-9071942, chosen for their high activity against both viruses, were tested for their ability to reduce SARS-CoV-2 and HCoV-229E infection. Dose-response assays showed that the effective dose for $50 \%$ of cells $\left(E D_{50}\right)$ values were $\sim 3 \mu \mathrm{M}$ and not significantly different between the selected inhibitors (Figure 1B, C). Pre-treatment of cells with the three compounds did not affect their corresponding antiviral activity (Supplementary figure 2). Selectivity Index (SI), was determined as cytotoxic concentration $\left(\mathrm{CC}_{50}\right)$ over $\mathrm{ED}_{50}$ values, in Huh-7.5.1 cells: T-1686568 was the most tolerated at $\mathrm{CC}_{50}>100 \mu \mathrm{M}$ (SARS-CoV-2 SI > 36) while P-7657632 and P-9071942 CC $_{50}$ values were $39 \mu \mathrm{M}$ (SARSCoV-2 SI=14) and $27 \mu \mathrm{M}$ (SARS-CoV-2 SI=8), respectively (Table 1). Thus, we decided to pursue T1686568 for further analysis. T-1686568 was shown to act synergistically with the FDA-approved antiviral Remdesivir in HCoV-229E infected cells. Using the Chou-Talalay method [18], an average Combination Index $(\mathrm{Cl})$ value of 0.7 (Figure 1D) was determined. $\mathrm{Cl}$ values $<1$ are defined as synergistic, $\mathrm{Cl}=1$ as additive, and $\mathrm{Cl}>1$ as antagonistic.

Relative inhibitor activity is often influenced by variability in different cell infection models [19]. While liver-derived Huh-7.5.1 cells were suitable for screening, initial validation, and toxicity studies, we performed a further investigation of T-1686568 potency in two other common SARS-CoV-2 infection models, colon Caco- 2 cells and the clinically-relevant lung Calu-3 cells. In all investigated cell lines, T1686568 provided a similar robust activity, with $\mathrm{ED}_{50}$ ranging from 4 to $7 \mu \mathrm{M}$ (Figure $2 \mathrm{~A}$ ). This observation indicates that GSK3 $\beta$ activity is needed for SARS-CoV-2 infection, independent of host cell type. These results demonstrated a reduction of intracellular viral markers. However, this may not directly translate to a reduction in viral particle production and release. We therefore measured the effect of T1686568 on the release of infective particles from SARS-CoV-2 infected Calu-3 cells and observed a significant 2-log reduction 48 hours after infection and treatment (Figure 2B).

Donor-derived organoids or induced pluripotent stem cell-derived organoids are the closest analogs to a patient in vivo model for SARS-CoV-2. These cells can be infected with SARS-CoV-2, have greater cell variability than monoclonal immortalized cell lines, and do not carry tumorigenic artefacts [20, 21]. We 
found that T-1686568 greatly decreased in dose dependent manner intracellular N protein levels in donor intestinal organoids infected with SARS-CoV-2 (Figure 2C).

Western blotting to detect SARS-CoV-2 proteins showed Spike S1 protein expression in infected Huh-7.5.1 cells was blocked by treatment with T-1686568, yet the non-structural protein (NSP2) and N protein could still be detected, with an apparent accumulation of the $\mathrm{N}$ protein (Figure 3 ). The specificity of the antibodies used to monitor the levels of the spike, NSP2 and N proteins was validated with dot blots with recombinant versions of these and other SARS-CoV-2 proteins and peptides (Figure 3).

Bioinformatic in silico and experimental data from multiple studies Mass Spec studies identified 37 phosphorylation sites in the $\mathrm{N}$ protein from coronaviruses (Supplemental Figure 3). At least 15 of these confirmed phosphorylation sites were located from Ser-176 to Ser-276 within the RS domain, and many of these conformed to consensus phosphosite recognition sequences for GSK3 [20]. Using in vitro kinase assays, we examined the ability of 9 different recombinant host kinases to phosphorylate a set of 8 SARS-CoV-2 nucleocapsid peptides that were patterned after the phosphosites in this region. Half of these peptides were prephosphorylated at sites that were predicted to seed subsequent GSK3-mediated phosphorylation. GSK3 $\beta$ further phosphorylated all four of these phosphopeptides, and PKCa was observed to be able to phosphorylate three of the four pairs of peptides provided that they were not prephosphorylated at the GSK3 priming phosphosites (Table 2). These in vitro phosphorylation assays showed that GSK3 $\beta$ phosphorylates more effectively peptides containing S180/S184, S190/S194 and T198/S202 that have already been primed (i.e. phosphorylated) in the flanking S188, T198 and S206 phosphosites, respectively. Both GSK3 $\beta$ and PKCa phosphorylated the full nucleocapsid (Table 3). These assays showed that both GSK3 $\beta$ and PKCa phosphorylate these peptides to varying degrees, but remarkably the combination of the two did not yield higher phosphorylation activity, likely due to incomplete priming of peptides by PKCa during the limited duration of the phosphotransferase assays (Table 3).

\section{Discussion}

Despite successful vaccine development, drugs against SARS-CoV-2 are still needed to manage active infections. Building on existing knowledge regarding the involvement of GSK3 $\beta$, we aimed at identification of compounds active against this key human kinase as potential coronaviridae inhibitors. Screening a targeted library provided a high rate of active compounds with greater than $50 \%$ inhibition of viral infection, with nearly half the compounds conferring some inhibitory effect against SARS-CoV-2 and HCoV-229E. Compared to the 'hit' rate of non-targeted host-directed therapy screens of $\sim 3 \%$ [22] [23] or $\sim 0.1 \%$ in non-specific screens [24], our results demonstrate a high benefit-to-investment ratio. More importantly, this screen demonstrates that more general host-kinase screens, which typically have 1-4 compounds representing each host kinase type, might be insufficient to rule-out specific kinases as potential targets. This is made evident through our study, as many GSK3 $\beta$ inhibitors in this library did not pass the $50 \%$ activity threshold. As such, a larger, focused screen might be a more comprehensive approach to determine the host-pathogen involvement of specific host kinases compared to general 
representative screens. Although beyond the scope of this study, targeted libraries also offer fascinating opportunities for further structure-function relationship studies.

Comparative analysis of SARS-CoV-2 and HCoV-229E screens suggests that viral infectivity reduction caused by GSK3 $\beta$ inhibition is not limited to SARS-CoV [6] and SARS-CoV-2, and thus may serve as a general target for development of drugs against various coronaviridae. Considering the timeline for new drug development and that approval is lengthy and continued coronavirus spread is likely, the ongoing COVID-19 pandemic necessitates exploratory development of such broad-spectrum antivirals. This is further supported by conserved arginine-serine rich domains, target regions for GSK3 phosphorylation, in $\mathrm{N}$ protein sequences across coronaviruses [7]. Despite limited $20-30 \%$ overall $\mathrm{N}$ protein sequence similarity, the domains maintain repeat motifs conducive for repeat phosphorylation [7].

Using SARS-CoV-2 nucleocapsid as a measurement of viral infection resulted in a robust $Z^{\prime}$ score for the screen $\left(Z^{\prime}=0.6\right)$ when compared alternatively to the dsRNA marker $\left(Z^{\prime}=0.3\right.$, supplementary Table 1$)$, likely due to ability of the antiviral dsRNA antibody to cross react with the host cell dsRNA. One viral marker may be insufficient to draw conclusions regarding viral infection, as in the case of GSK3 $\beta$, due to potential target-marker interactions. Although treated cells do accumulate nucleocapsid, we found a strong agreement between nucleocapsid and dsRNA marker levels in their response to the inhibitors tested, suggesting that in this high-content screening assay, nucleocapsid can be used as a marker for viral infection. Screen inhibition readouts were similar (mean difference of $10 \%$ ) to those in nucleocapsid expression. Media-released virus measured using plaque assay quantification confirmed that the inhibitory effect of GSK3ß-inhibition extends to SARS-CoV-2 assembly and maturation.

T-1686568 was selected due to its high selectivity index. Similar to the validation of viral infection measurements using different viral markers, utilising different cell lines is important for any robust immortalized cell line screen, and particularly for compound activity against SARS-CoV-2, given the many reporting mismatches of screen results between different cell lines [19]. To guard against this, we internally validated our screen through the use of different cell lines and found a consistent T-1686568 inhibitory effect. Of particular note, host-targeting inhibitors designed against viral entry mechanisms, such as ACE2 and TMPRSS2, present higher variability in the expression of these host factors between cell lines [25]. Our observations suggest GSK3 $\beta$ is a conserved pathway critical to SARS-CoV-2 infection in many tissues, and strongly recommend it be considered in the selection of targets for drug development. This is further supported through the observation of SARS-CoV-2 inhibition in patientderived colon organoids, which contain multiple different types of infectible cells [26].

Mass spectrometry studies have demonstrated that the RS domain of the $\mathrm{N}$ protein that encompasses residues Ser-176 to Ser-276 undergoes extensive phosphorylation (Supplemental Figure 3). Using synthetic peptides modeled after these phosphosites, we have shown that they can be directly phosphorylated by recombinant GSK3 $\beta$, provided that these peptides are prephosphorylated at priming sites. Recombinant PKC-alpha was able to phosphorylate several of the priming sites for the GSK3 $\beta$ in vitro, although other kinases may be responsible for this in vivo. Our findings complement the recent in 
vivo studies of Liu et al. [7], who showed that GSK3 acted upstream of the N protein to mediate its phosphorylation, and by site-directed mutagenesis determined that the suspected Ser-188 and Ser-206 priming phosphosites for GSK3 recognition were required for phosphorylation-dependent mobility shifts of the N protein on SDS-PAGE gels by Western blotting. Thr-205 may also act a priming site for phosphorylation of the Ser-201 and Ser-197 sites that are known to be phosphorylated in cells and predicted for GSK3 targeting as well.

The SARS-CoV-2 N protein is an abundant RNA-binding protein critical for viral genome packaging, yet the molecular processes and characteristics of this function are not fully understood. The $\mathrm{N}$ protein is a phosphoprotein that associates with the viral RNA genome to form the ribonucleoprotein core [27]. The $\mathrm{N}$ protein contains three dynamic disordered regions that house putative transiently-helical binding motifs. The two folded domains interact minimally such that full-length $\mathrm{N}$ protein is a flexible and multivalent RNA-binding protein.

The phosphorylation of the RS domain in SARS-CoV and SARS-CoV-2 has been implicated in the regulation of $\mathrm{N}$ protein binding to RNA, multimerization and subcellular location [28-32] Studies with SARS-CoV-2 indicate that changes in the phosphorylation status of the RS domain induces profound alterations in the association of multiple nucleocapsid proteins with a single viral RNA in a structured oligomer with RNA-protein and protein-protein interactions to switch to one that permits more viral genome processing [33]. Following N protein phosphorylation in the RS domain, the RNA-protein complex is able to recruit the stress granule protein G3BP1 and suppress the G3BP-dependent host immune response [34]. In our study, the blockage of N protein phosphorylation by GSK3 appeared to result in partial accumulation of the $\mathrm{N}$ protein, and completely prevented spike protein production to allow formation of the virus particles.

Several recent studies $[33,35-40]$ have shown that the $\mathrm{N}$ protein also undergoes liquid-liquid phase separation when mixed with RNA, and polymer theory predicts that the same multivalent interactions that drive phase separation also engender RNA compaction. Our future studies are geared toward validating our hypothesis that phosphorylation of the SARS-CoV-2 N protein by GSK3 $\beta$ is needed for forming a physiological 'phase separation' state required for the N protein / RNA assembly and budding.

Interestingly, evidence in the literature suggest that the spike protein is also a target for phosphorylation [41] that may affect its expression level and cellular trafficking. Coincidently, we noticed that the reduced levels of the Spike protein S1 subunit (Figure 3) are consistent with T-1686568 induced decrease of the viral titer. Together, our findings strongly support inhibition of GSK3 as an effective anti-viral strategy for treatment of COVID-19 and other coronaviruses at their earliest stages.

\section{Materials And Methods}

\section{Cell culture}


Caco-2 cells (ATCC $\circledast$ HTB-37 $7^{\text {TM }}$ ), Calu-3 cells (ATCC $\circledast$ HTB-55 $5^{\text {TM }}$ ) and Vero E6 cells (ATCC $\circledast$ CRL-1586 $6^{\text {TM }}$ ) were cultivated in accordance with ATCC recommendations. Human hepatoma, Huh-7.5.1 cells [42] were obtained from APATH LLC and cultivated in Dulbecco's modified Eagle's medium (DMEM) supplemented with $10 \%$ fetal bovine serum, $0.1 \mathrm{mM}$ nonessential amino acids, $1 \mathrm{mM}$ sodium pyruvate, and $10 \mathrm{mM}$ HEPES. Experiments were performed in Huh-7.5.1 and Vero E6 cells below passage 30, and Caco-2 and Calu- 3 cells below passage 6 . All cells were expanded in T75 flask with a with $5 \%$ carbon dioxide at $37^{\circ} \mathrm{C}$. Cell density was kept between 0.25 and 2 million cells $/ \mathrm{mL}$. Intestinal biopsy-derived colonoids from healthy donors were donated from the Johns Hopkins Conte Digestive Disease Basic and Translational Research Core Center (NIH NIDDK P30-DK089502) and grown based using methods by Staab et al [43].

\section{Compounds}

Compounds were kindly supplied by Takeda Pharmaceuticals, solubilized in $100 \%$ dimethyl sulfoxide (DMSO, Sigma) in a concentration of $10 \mathrm{mM}$ and stored at $-20^{\circ} \mathrm{C}$. Compounds were diluted in cell-specific media prior to treatment, limited to less than 5 freeze-thaw cycles for each aliquot.

\section{Viral Infection Assays}

All SARS-CoV-2 infections were carried out in a Biological Contamination Level 3 facility (UBC FINDER) in accordance with Public Health Agency of Canada and UBC FINDER regulations. SARS-CoV-2 SB2 was isolated by Dr. Samira Mubareka (Sunnybrook, ON Canada) [44] and passaged in Vero E6 cells. For experiments, passage 3 of the virus was used with a determined viral titer of $1.5 \times 10^{7}$ plaque forming units (PFU)/mL. HCoV-229E was kindly obtained from Dr. Eric Jan, (UBC, Canada) and infections were caried out in Biological Contamination Level 2+ laboratory in Huh-7.5.1 cells at $33^{\circ} \mathrm{C}$. Cells were seeded at a concentration of 10,000 cells/well in $96-$ well plates, $24 \mathrm{~h}$ prior to infection. SARS-CoV-2 stocks were diluted in cell-specific media to a Multiplicity of Infection (MOI) of 1 for Caco-2, Vero E6, and Huh-7.5.1, and $\mathrm{MOI}$ of 2 for Calu- 3 cells and colonoids. Cells were pre-treated with compounds for 3 hours and then incubated with the virus for 2 days (colonoids - 3 days), followed by fixation of the cells with $3.7 \%$ formalin for 30 minutes for immunostaining and to inactivate the virus. The fixative was removed, and washed with PBS, followed by immunostaining of the cells with the mouse primary antibody J2 (dsRNA; Jena Biosciences) and rabbit primary antibody HL344 (SARS-CoV-2 nucleocapsid; GeneTex) at working dilutions of 1:1000 for $1 \mathrm{~h}$ at room temperature. Secondary antibodies were used at a 1:2000 dilution and included the goat-anti mouse IgG Alexa Fluor 488 (cat \#A11001) and goat-anti rabbit IgG Alexa Fluor 555 (A21428) from Invitrogen with the nuclear stain Hoechst 33342 at $1 \mu \mathrm{g} / \mathrm{mL}$ for $1 \mathrm{~h}$ in room temperature in the dark. After antibody removal, plates were kept covered in aluminum foil until scanning to avoid photobleaching.

\section{High content screening methodology and parameters}

Monitoring of the total number of cells (based on nuclei staining) and number of virus infected cells (based on dsRNA and Nucleocapsid staining) was performed using the Celllnsight CX5 High Content Screening platform (Thermo Fisher), as previously described [23]. Shortly, nuclei are identified and counted using the $350 / 461 \mathrm{~nm}$ wavelength (Hoechst 33342); cell debris and other particles are deducted 
based on a size filter tool. A Region of Interest (ROI, or "circle") is then drawn around each host cell and validated against the bright field image to correspond with host cell membranes. The ROI encompasses the "spots", where dsRNA (485/521 nm wavelength) and SARS-CoV-2 nucleocapsid (533/588 nm wavelength) are localized. Finally, the software (HCS Studio Cell Analysis Software, version 4.0) identifies, counts and measures the pixel area and intensity of the "spots" within the "circle". The fluorescent spot area measured within each cell (circle) is then added and quantified for each well. The total circle spot intensity of each well corresponds to the viral load, and is normalized to non-infected cells, and to cells infected with no compound. Nuclei stain (Hoechst 33342) was used to quantify cell loss (due to cytotoxicity or loss of adherence), and to normalize the changes in viral load resulting from a decrease in cell numbers.

\section{Dose-response analysis}

Intracellular dose-response of viral infection (dsRNA or Nucleocapsid signal) in the presence of compounds was performed at dilution factors of 1:1 with 3 technical replicates in each experiment, and at least 3 biological replications per compound concentration. Viral infection levels were interpolated to negative control $(0.1 \%$ DMSO, no infection $)=0$, and positive control $(0.1 \%$ DMSO, with infection $)=100$. GraphPad Prism $9^{\text {TM }}$ (GraphPad Software, Inc.) non-linear regression fit modeling variable slope was used to generate a dose-response curve $\left(Y=B o t t o m+(\right.$ Top-Bottom $) /\left(1+10^{\wedge}((\right.$ LoglC50-X)*HillSlope $))$, constrained to top $=100$, bottom $=0$.

\section{Sources of recombinant proteins, peptides and antibodies}

The following recombinant SARS-CoV-2 proteins that were expressed in E. coli were procured from the MRC Protein Phosphorylation and Ubiquitination Unit Reagents and Services at the University of Dundee (Dundee, Scotland) myelin basic protein-spike receptor binding domain (MBP-Spike RBD) amino acids (aa) 319-541 (DU 67753); glutathione S-transferase-nonstructural protein-1 (GST-NSP1) aa 1-180 (DU 66413); GST-NSP2 aa 1-638 (DU 66414); NSP13 aa 1-601 (DU 66417); GST-NSP14 aa 1-527 (DU 66418); GST-NSP15 aa 1-527 (DU 66419); GST-membrane protein aa 1-222 (DU 67699); and GST-nucleocapsid protein aa 1-419 (DU 67726). Additional recombinant SARS-CoV-2 proteins were produced as described [35]. This included fusion proteins with a carrier protein module, a thermophilic family 9 carbohydratebinding module 208 (CBM9).

All of the protein kinases used in this study were active preparations of recombinant, GST-fusion human proteins expressed in E. coli or Sf9 insect cell, and sourced from SignalChem (Richmond, BC, Canada). The kinases tested were cyclin-dependent kinase 2/cyclin A2 (CDK2/A2; C29-10G), casein kinase 1-alpha1 (CK1a1; C64-10G), casein kinase 1-delta (CK181; C65-10G), casein kinase 1-gamma-1 (CK1 Y1; C6811G), casein kinase 2-alpha-1 (CK2a1; C70-10G), Extracellularly regulated kinase-1 (ERK1; M29-10G), glycogen synthase kinase-3-beta (GSK3ß; G09-10G), cAMP-dependent protein kinase catalytic subunitalpha (PKACa; P51-10G), and protein kinase C-alpha (PKCa; P61-18G). Production of all synthetic peptides patterned after the sequences in the SARS-CoV-2 proteins was performed by Lifetein (Somerset, NJ, USA). 
Peptide affinity-purified rabbit polyclonal antibodies directed against synthetic peptides based on the following SARS-CoV-2 proteins were used from Kinexus Bioinformatics: Nucleocapsid aa 156-170 (NNCOV2N-1); ORF1a aa 735-750 - NSP2 (NNCOV2-1A-2); and Spike aa 574-588 (NNCOV2S-10).

\section{Protein kinase reactions}

All protein kinase reactions were performed with the ADP-Glo Kinase Assay from ProMega (Madison, WI, USA). Substrate peptides were assayed at a final concentration of $\sim 250 \mu \mathrm{M}$ with $250 \mu \mathrm{M}$ ATP for 30 min at $30^{\circ} \mathrm{C}$ in a final volume of $25 \mu \mathrm{l}$. Recombinant nucleocapsid was similarly assayed at a final concentration of $13.4 \mathrm{ng} / \mu \mathrm{l}$. The concentrations of recombinant protein kinases used was $\sim 2 \mathrm{ng} / \mu \mathrm{l}$.

\section{Western blotting}

To investigate protein expression levels, infected cells were lysed by scraping into lysis buffer (20 mM 3( $N$-morpholino) propanesulfonic acid [MOPS], pH 7.2, 5 mM EDTA, 2 mM ethylene glycol tetraacetic acid [EGTA], 0.5\% [vol/vol] Triton X-100, $30 \mathrm{mM} \mathrm{NaF,} 20 \mathrm{mM} \mathrm{Na}_{4} \mathrm{P}_{2} \mathrm{O}_{7}, 1 \mathrm{mM} \mathrm{Na}_{3} \mathrm{VO}_{4}, 40 \mathrm{mM} \beta$ glycerophosphate, $1 \mathrm{mM}$ dithiothreitol [DTT], $1 \mathrm{mM}$ phenylmethylsulfonyl fluoride, $3 \mathrm{mM}$ benzamidine, 5 $\mu \mathrm{M}$ pepstatin $\mathrm{A}$, and $10 \mu \mathrm{M}$ leupeptin and $0.5 \mu \mathrm{M}$ Aprotonin, $0.5 \mathrm{mM}$ Tris (2-carboxyethyl) phosphine hydrochloride (TCEP)- pH- 9.0. Cells were briefly sonicated, 2-Nitro-5-thiocyanatobenzoic acid (NTCB) was added to a final concentration of $6 \mathrm{mM}$. Tubes were rotated to thoroughly expose tube's internals to lysis buffer, and samples were frozen at $-20^{\circ} \mathrm{C}$. Similarly-treated infected cells were tested for plaque formation using a standard PFU test, to confirm lysis buffer deactivated viral particles prior to removal of the assay tubes from UBC FINDER. Incubation was performed in a $37^{\circ} \mathrm{C}$ water bath for $15 \mathrm{~min}$ and then subjected to ultracentrifugation at $100,000 \times g$ for $30 \mathrm{~min}$. The resulting samples were resolved by SDS-PAGE and transferred onto a nitrocellulose membrane. The blot was probed with the designated antibodies and developed by ECL.

\section{Declarations}

\section{Acknowledgments:}

Financial support was provided by the TB Veterans Association (YA) and Genome British Columbia / COVID-19 Rapid Response Funding Initiative (FJ). We thank Dr. Samira Mubareka and Dr. Arinjay Banerjee, (Sunnybrook Health Sciences Centre and Research Institute, University of Toronto) for providing the SARS-CoV-2 (SARS-COV-2/Canada/VIDO-01/2020). We further thank Dr. Alex Ball, Jr., MD, Senior Scientist (Genetex), for supplying the SARS-CoV-2 (COVID-19) nucleocapsid antibody [HL344] (GTX635679). We thank Dr. Nicholas Zachos from John Hopkins University for kindly providing human colonoids and to the patients who donated these samples for research. The authors acknowledge the support of the CL-3 facility (Facility for Infectious Disease and Epidemic Research (FINDER) of the Life Sciences Institute of the University of British Columbia. We would like to acknowledge BIO Ventures for Global Health (BVGH) for facilitating a collaboration with Takeda Pharmaceuticals through WIPO 
Re:Search for the sharing of GSK3 inhibitors. We also thank Dr. Andrea Olmstead for proofreading the manuscript.

\section{Conflicts of Interest:}

$\mathrm{SP}$ is the majority shareholder of Kinexus Bioinformatics Corporation. All other authors declare no conflicts.

\section{AUTHOR CONTRIBUTIONS}

Conceptualization, T.S., S.P., and Y.A-G.; Methodology, T.S., C.R., D.F.H.W., I.S., S.P., T.St., W.R., F.J., S.P. and Y.A-G.; Investigation, T.S., C.R., S.P., V.P., and W.R.; Writing - Original Draft, T.S., C.R., V.P., S.P., and Y.A-G. ; Writing - Review \& Editing, T.S., C.R., V.P., S.P., and Y.A-G. Funding Acquisition, F.J., S.P., and Y.A.-G.; Resources, S.P, F.J., T.St., and Y.A.-G.; Supervision, F.J., TS.t., S.P., and Y.A.-G.

\section{References}

1. John Hopkins Corona Viruse Resource Center. 2021; Available from: https://coronavirus.jhu.edu/map.html.

2. Chen, J., et al., Epidemiological and economic impact of COVID-19 in the US. Sci Rep, 2021. 11(1): p. 20451.

3. Sen, P., et al., Burden and characteristics of COVID-19 in the United States during 2020. Nature, 2021. 598(7880): p. 338-341.

4. Gordon, D.E., et al., A SARS-CoV-2 protein interaction map reveals targets for drug repurposing. Nature, 2020. 583(7816): p. 459-468.

5. Coleman, C.M., et al., Abelson Kinase Inhibitors Are Potent Inhibitors of Severe Acute Respiratory Syndrome Coronavirus and Middle East Respiratory Syndrome Coronavirus Fusion. J Virol, 2016. 90(19): p. 8924-33.

6. Wu, C.H., et al., Glycogen synthase kinase-3 regulates the phosphorylation of severe acute respiratory syndrome coronavirus nucleocapsid protein and viral replication. J Biol Chem, 2009. 284(8): p. 5229-39.

7. Liu, X., et al., Targeting the coronavirus nucleocapsid protein through GSK-3 inhibition. Proc Natl Acad Sci U S A, 2021. 118(42). 
8. Rana, A.K., et al., Glycogen synthase kinase-3: A putative target to combat severe acute respiratory syndrome coronavirus 2 (SARS-CoV-2) pandemic. Cytokine Growth Factor Rev, 2021. 58: p. 92-101.

9. Embi, N., D.B. Rylatt, and P. Cohen, Glycogen synthase kinase-3 from rabbit skeletal muscle.

Separation from cyclic-AMP-dependent protein kinase and phosphorylase kinase. Eur J Biochem, 1980. 107(2): p. 519-27.

10. Tung, H.Y., et al., The catalytic subunits of protein phosphatase- 1 and protein phosphatase $2 A$ are distinct gene products. Eur J Biochem, 1984. 138(3): p. 635-41.

11. Goode, N., et al., Differential regulation of glycogen synthase kinase-3 beta by protein kinase $C$ isotypes. J Biol Chem, 1992. 267(24): p. 16878-82.

12. Barrell, W.B., H.L. Szabo-Rogers, and K.J. Liu, Novel reporter alleles of GSK-3alpha and GSK-3beta. PLoS One, 2012. 7(11): p. e50422.

13. Plyte, S.E., et al., Glycogen synthase kinase-3: functions in oncogenesis and development. Biochim Biophys Acta, 1992. 1114(2-3): p. 147-62.

14. Cohen, P. and S. Frame, The renaissance of GSK3. Nat Rev Mol Cell Biol, 2001. 2(10): p. 769-76.

15. Saraswati, A.P., et al., Glycogen synthase kinase-3 and its inhibitors: Potential target for various therapeutic conditions. Eur J Med Chem, 2018. 144: p. 843-858.

16. Wang, H., et al., GSK3beta and the control of infectious bacterial diseases. Trends Microbiol, 2014. 22(4): p. 208-17.

17. Saitoh, M., et al., Design, synthesis and structure-activity relationships of 1,3,4-oxadiazole derivatives as novel inhibitors of glycogen synthase kinase-3beta. Bioorg Med Chem, 2009. 17(5): p. 2017-29.

18. Chou, T.C. and P. Talalay, Quantitative analysis of dose-effect relationships: the combined effects of multiple drugs or enzyme inhibitors. Adv Enzyme Regul, 1984. 22: p. 27-55.

19. Kumar, S., et al., Clinically relevant cell culture models and their significance in isolation, pathogenesis, vaccine development, repurposing and screening of new drugs for SARS-CoV-2: a systematic review. Tissue Cell, 2021. 70: p. 101497.

20. Mulay, A., et al., SARS-CoV-2 infection of primary human lung epithelium for COVID-19 modeling and drug discovery. bioRxiv, 2020.

21. Han, Y., et al., Identification of SARS-CoV-2 inhibitors using lung and colonic organoids. Nature, 2021. 589(7841): p. 270-275.

22. Han, H.W., et al., Drug Discovery Platform Targeting M. tuberculosis with Human Embryonic Stem Cell-Derived Macrophages. Stem Cell Reports, 2019. 13(6): p. 980-991.

23. Shapira, T., et al., High-Content Screening of Eukaryotic Kinase Inhibitors Identify CHK2 Inhibitor Activity Against Mycobacterium tuberculosis. Front Microbiol, 2020. 11: p. 553962.

24. Pethe, K., et al., Discovery of Q203, a potent clinical candidate for the treatment of tuberculosis. Nat Med, 2013. 19(9): p. 1157-60.

25. Zecha, J., et al., Data, Reagents, Assays and Merits of Proteomics for SARS-CoV-2 Research and Testing. Mol Cell Proteomics, 2020. 19(9): p. 1503-1522. 
26. Shapira, T., et al., A novel highly potent inhibitor of TMPRSS2-like proteases blocks SARS-CoV-2 variants of concern and is broadly protective against infection and mortality in mice. bioRxiv, 2021.

27. Chang, C.K., et al., Modular organization of SARS coronavirus nucleocapsid protein. J Biomed Sci, 2006. 13(1): p. 59-72.

28. Nelson, G.W., S.A. Stohlman, and S.M. Tahara, High affinity interaction between nucleocapsid protein and leader/intergenic sequence of mouse hepatitis virus RNA. J Gen Virol, 2000. 81(Pt 1): p. 181-8.

29. Chen, $\mathrm{H}$., et al., Mass spectroscopic characterization of the coronavirus infectious bronchitis virus nucleoprotein and elucidation of the role of phosphorylation in RNA binding by using surface plasmon resonance. J Virol, 2005. 79(2): p. 1164-79.

30. Peng, T.Y., K.R. Lee, and W.Y. Tarn, Phosphorylation of the arginine/serine dipeptide-rich motif of the severe acute respiratory syndrome coronavirus nucleocapsid protein modulates its multimerization, translation inhibitory activity and cellular localization. FEBS J, 2008. 275(16): p. 4152-63.

31. Stohlman, S.A., et al., Synthesis and subcellular localization of the murine coronavirus nucleocapsid protein. Virology, 1983. 130(2): p. 527-32.

32. Surjit, M., et al., The severe acute respiratory syndrome coronavirus nucleocapsid protein is phosphorylated and localizes in the cytoplasm by 14-3-3-mediated translocation. J Virol, 2005. 79(17): p. 11476-86.

33. Carlson, C.R., et al., Phosphoregulation of Phase Separation by the SARS-CoV-2 N Protein Suggests a Biophysical Basis for its Dual Functions. Mol Cell, 2020. 80(6): p. 1092-1103 e4.

34. Lu, S., et al., The SARS-CoV-2 nucleocapsid phosphoprotein forms mutually exclusive condensates with RNA and the membrane-associated M protein. Nat Commun, 2021. 12(1): p. 502.

35. Cubuk, J., et al., The SARS-CoV-2 nucleocapsid protein is dynamic, disordered, and phase separates with RNA. Nat Commun, 2021. 12(1): p. 1936.

36. Iserman, C., et al., Genomic RNA Elements Drive Phase Separation of the SARS-CoV-2 Nucleocapsid. Mol Cell, 2020. 80(6): p. 1078-1091 e6.

37. Perdikari, T.M., et al., SARS-CoV-2 nucleocapsid protein phase-separates with RNA and with human hnRNPs. EMBO J, 2020. 39(24): p. e106478.

38. Roden, C.A., et al., Double-stranded RNA drives SARS-CoV-2 nucleocapsid protein to undergo phase separation at specific temperatures. bioRxiv, 2021.

39. Savastano, A., et al., Nucleocapsid protein of SARS-CoV-2 phase separates into RNA-rich polymerasecontaining condensates. Nat Commun, 2020. 11(1): p. 6041.

40. Wang, S., et al., Targeting liquid-liquid phase separation of SARS-CoV-2 nucleocapsid protein promotes innate antiviral immunity by elevating MAVS activity. Nat Cell Biol, 2021. 23(7): p. 718732.

41. Ord, M., I. Faustova, and M. Loog, The sequence at Spike S1/S2 site enables cleavage by furin and phospho-regulation in SARS-CoV2 but not in SARS-CoV1 or MERS-CoV. Sci Rep, 2020. 10(1): p. 16944. 
42. Zhong, J., et al., Robust hepatitis C virus infection in vitro. Proc Natl Acad Sci U S A, 2005. 102(26): p. 9294-9.

43. Staab, J.F., et al., Co-Culture System of Human Enteroids/Colonoids with Innate Immune Cells. Curr Protoc Immunol, 2020. 131(1): p. e113.

44. Banerjee, A., et al., Isolation, Sequence, Infectivity, and Replication Kinetics of Severe Acute Respiratory Syndrome Coronavirus 2. Emerg Infect Dis, 2020. 26(9): p. 2054-2063.

\section{Tables}

The tables can be found in the supplementary files section.

\section{Figures}

A

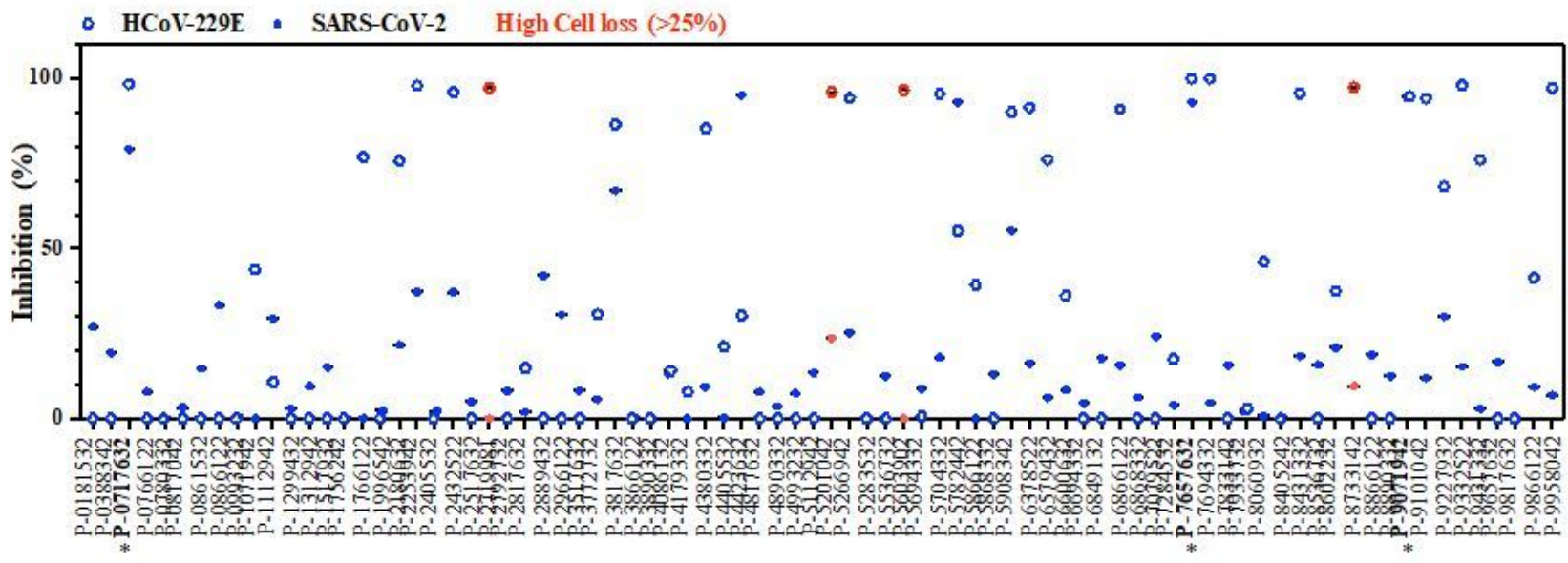

B SARS-CoV-2::Huh-7.5.1

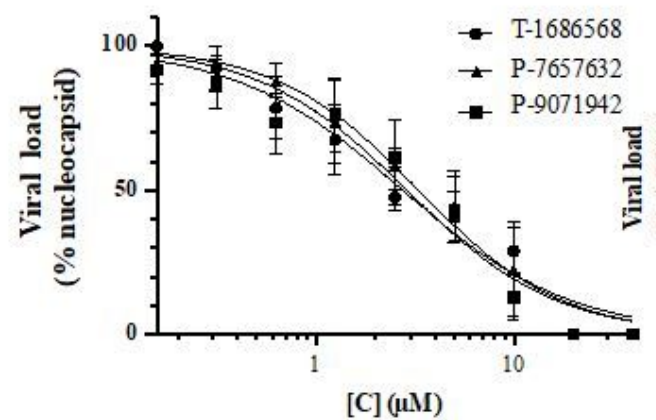

C

HCoV-229E::Huh-7.5.1

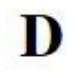

D

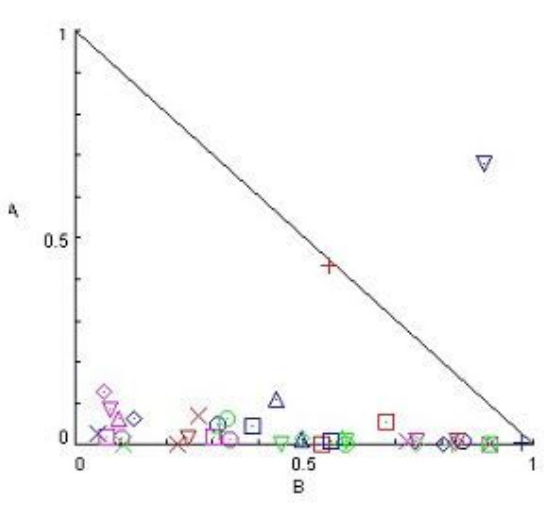

Figure 1

(A) High-Content Screen of SARS-CoV-2 (full circles) and HCoV-229E (empty circles) infected Huh-7.5.1 cells with the Takeda GSK3 $\beta$-focused library at a single dose of $10 \mu \mathrm{M}$. Inhibition was interpolated to both a non-infected control and an infected, untreated control, quantifying intracellular $\mathrm{N}$ protein. Cell loss was 
assessed to avoid quantifying small populations, and compounds resulting in $>25 \%$ cell loss are marked in red. Compounds marked with an asterisk were further pursued. The $Z^{\prime}$ of two independent screens were 0.54 and 0.74 for SARS-CoV-2, and 0.68 for HCoV-229E. (B-C) Dose-response validation of compounds of interest. Each dose is an average of four independent experiments, error bars show SEM. Viral load of SARS-CoV-2 (B) was determined by measuring intracellular N protein, and viral load of HCoV-229E (C) by measuring dsRNA. (D) Representative synergy isoblogram of T-1686568 and Remdesivir analyzed with HCoV-229E.

A $\quad$ B

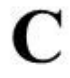

SARS-CoV-2::Calu-3

P-0717632

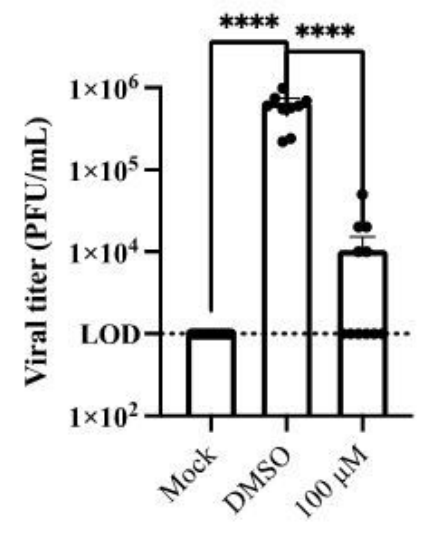

SARS-CoV-2::Immortalized cell lines P-0717632

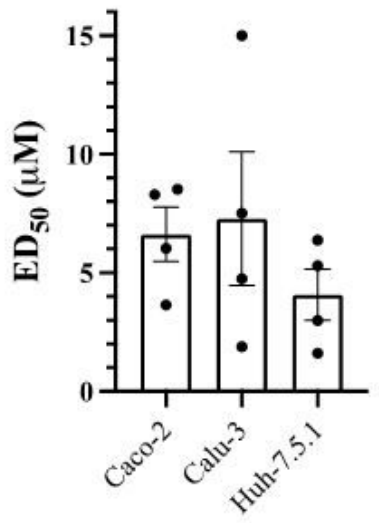

SARS-CoV-2::Colonoids P-0717632

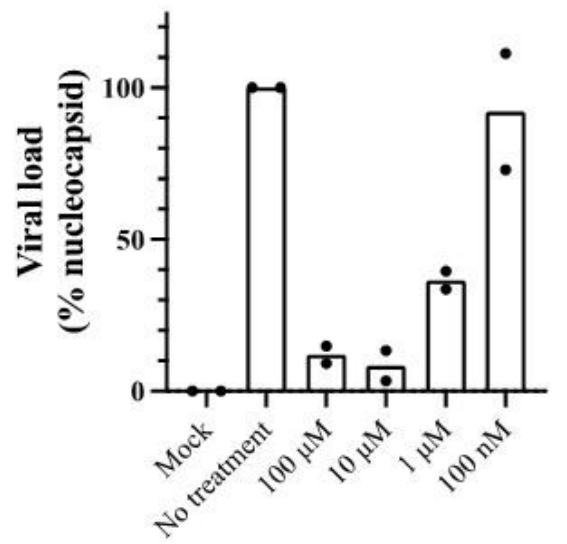

Figure 2

(A) Dose-response inhibition effect of T-1686568 in three infected immortalized cell lines. Data from four independent experiments; error bars are the SEM. (B) Viral titer from 48-hour infected Calu-3 cells, with $(100 \mu \mathrm{M})$ and without (DMSO) T-1686568 from 12 independent infections; error bars are the SEM. Oneway ANOVA with Bonferroni multiple comparison correction, $P<0.0001$. LOD - Limit of Detection. (C) Semi-dose-response inhibition effect of T-1686568 in primary donor-derived colon organoids, 72-hours post infection. Viral load interpolated to both non-infected cells (mock) control and an infected, untreated control, quantifying intracellular $\mathrm{N}$ protein. 
Nucleocapsid antibody (aa 156-170)
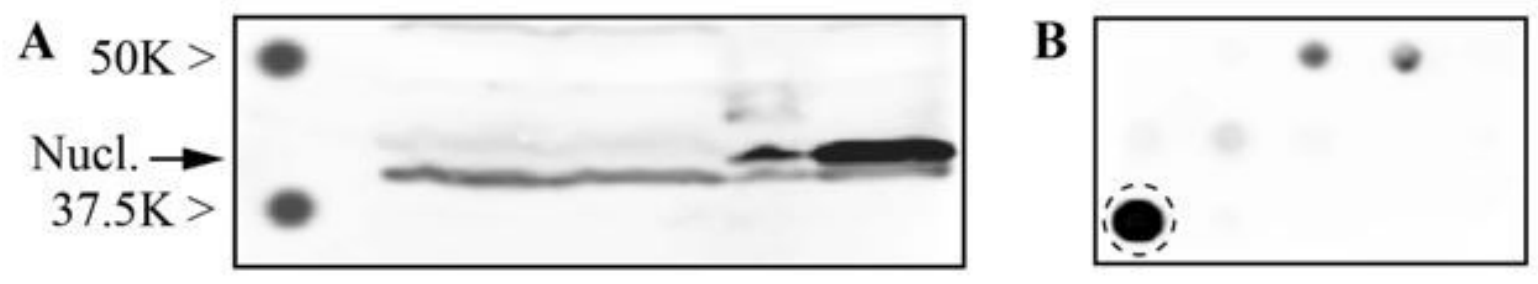

ORF1a: NSP2 antibody (aa 735-750)
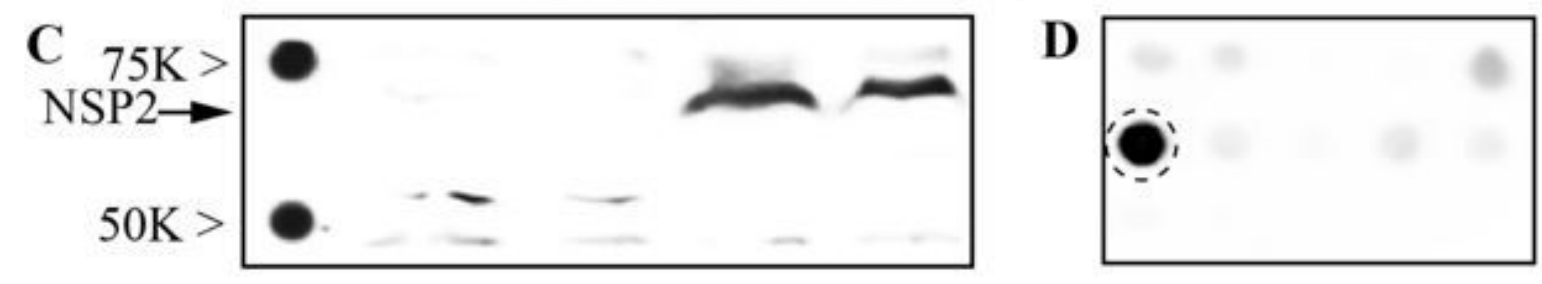

Spike antibody (aa 574-588)
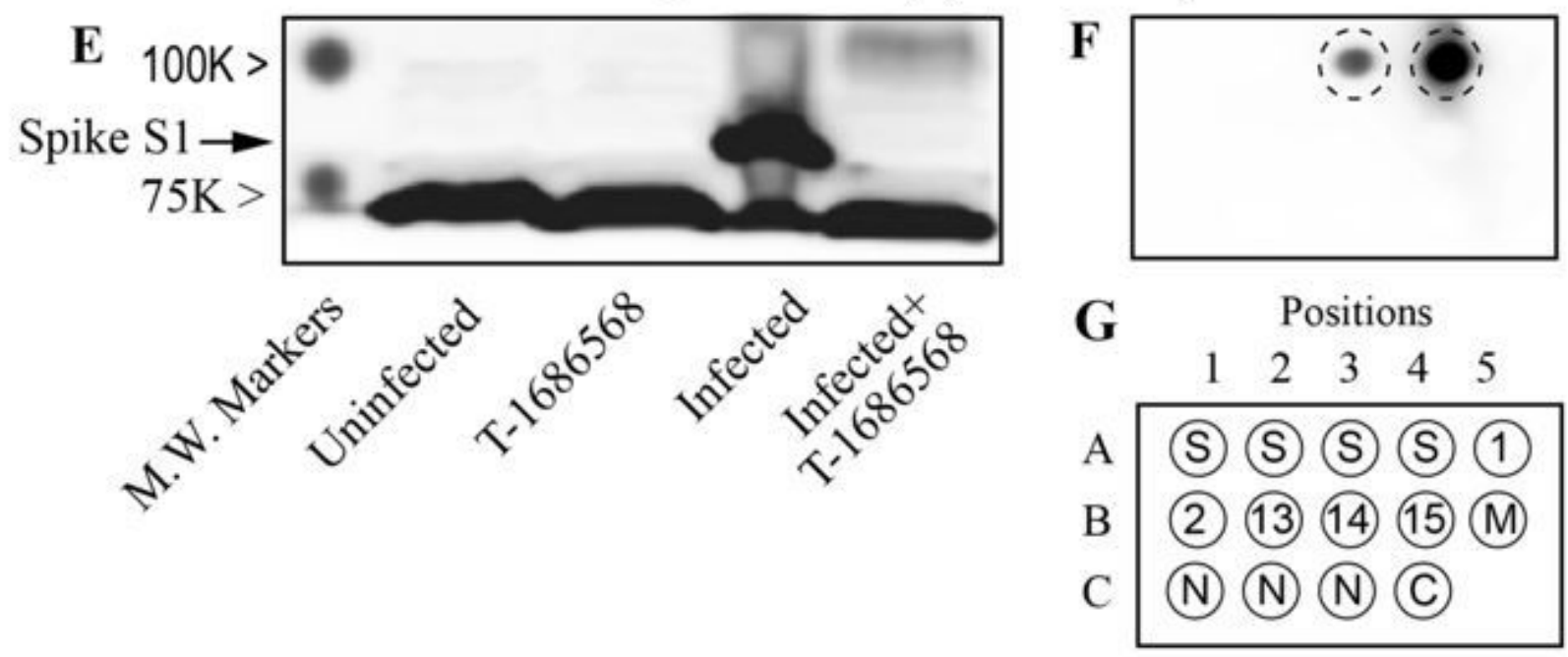

Al - MBP-Spike protein RBD aa 319-541

A2 - ID-F - Spike protein aa 450-469

A3 - ID-H1 - Spike protein aa 552-602

A4 - ID-H1 - Spike protein aa 552-602

A5 - GST-NSP1 protein aa 1-180

B1 - GST-NSP2 protein aa 1-638

B2 - NSP13 protein aa 1-601

B3 - GST-NSP14 protein aa 1-527
B4 - GST-NSP15 protein aa 1-527

B5 - GST-Membrane protein aa 1-222

$\mathrm{Cl}$ - Nucleocapsid protein aa 1-419

C2 - CBD9-Nucleocapsid protein aa 386-403

C3 - CBD9-Nucleocapsid protein aa 386-403

C4 - CBD9 only

C5 - Unspotted blank

\section{Figure 3}

Presence of SARS-CoV-2 proteins in infected Huh-7.5.1 cells treated with and without T-1686568. (A, C, E) Cells were pre-treated with $10 \mu \mathrm{M} \mathrm{T}-1686568$ for 3 hours and then incubated with the virus for 2 days prior to harvesting and western blotting. (B, D, F) Recombinant preparations of SARS-CoV-2 protein and peptides were robotically spotted on to nitrocellulose membranes as configured in ( $G$ ) and then immunoblotted with the following antibodies that were generated against peptides patterned after 
sequences in: (A, B) the nucleocapsid (Nucl.; NNCOV2N-1 raised against amino acids 156-170); (C,D). the non-structural protein NSP2 in the ORF1; NNCOV2-1A-2 raised against amino acids 735-750); and the spike protein S1 subunit (Spk S1; NNCOV2S-10 raised against amino acids 574-588). Locations of expected positions of target proteins and peptides are circled in the dot blots shown. Examples shown are representative of triplicate experiments.

\section{Supplementary Files}

This is a list of supplementary files associated with this preprint. Click to download.

- SuplementalFigures.docx

- sourcedatascreening.xlsx

- SupplementaryTable1.xlsx

- table3.jpg

- table2.jpg

- table1.jpg 